Relations industrielles

Industrial Relations

Nouveau manuel de l'agent de maîtrise, par Pierre Demarne et Jacques Ferras, Entreprise Moderne d'Édition, Paris, 1967, 157 pages.

\title{
Rosaire Roy
}

\section{Volume 23, numéro 1, 1968}

URI : https://id.erudit.org/iderudit/027882ar

DOI : https://doi.org/10.7202/027882ar

Aller au sommaire du numéro

\section{Éditeur(s)}

Département des relations industrielles de l'Université Laval

\section{ISSN}

0034-379X (imprimé)

1703-8138 (numérique)

Découvrir la revue

\section{Citer ce compte rendu}

Roy, R. (1968). Compte rendu de [Nouveau manuel de l'agent de maîtrise, par Pierre Demarne et Jacques Ferras, Entreprise Moderne d’Édition, Paris, 1967, 157 pages.] Relations industrielles / Industrial Relations, 23(1), 182-183.

https://doi.org/10.7202/027882ar

Tous droits réservés (C) Département des relations industrielles de l'Université Laval, 1968
Ce document est protégé par la loi sur le droit d'auteur. L'utilisation des services d'Érudit (y compris la reproduction) est assujettie à sa politique d'utilisation que vous pouvez consulter en ligne.

https://apropos.erudit.org/fr/usagers/politique-dutilisation/ 
tion de la «nouvelle classe ouvrière » et sur l'évolution du * monde des employés de bureau », il est remarquable que le « malaise des cadres » attire oujourd'hui l'attention aussi bien des sociologues que des acteurs sociaux.

Les cadres apparaissent comme un enjeu pour les forces sociales en présence. Les dirigeants de l'industrie s'inquiètent de ce qu'ils considèrent comme un offaiblissement de la loyauté des cadres à l'égard de l'entreprise et de leur allégeance à la direction. Ii est vrai que lors de récents conflits sociaux des cadres sont pafois sortis de leut traditionnelle neutralité, prenant position en face de la direction, et allant jusqu'à se solidariser avec les techniciens ou les ouvriers. Constatant que la place des cadres entre le monde patronal et le monde ouvrier évolue, les dirigeants se demandent alors comment les intégrer à l'entreprise et comment renforcer parmi eux la légitimité de leur autorité. Les responsables des organisations syndicales de leur côté attentifs à cette évolution, cherchent à la canaliser et à l'orienter, conscients de l'importance que représentent, avec les techniciens, les ingénieurs et les cadres pour l'avenir du mouvement syndical; la tendance récente à une syndicalisotion accrue de ces catégories de salariés ne fait que confirmer leur volonté de les organiser.

Enfin, l'Etat lui-même ne peut rester indifférent devant une telle évolution; les codres ne représentent pas seulement pour lui un ensemble de problèmes à résoudre dont les plus actuels sont ceux du a recyclage " et du chômage, mais aussi une force sociale avec laquelle il doit désormais compter. Sans doute, le temps n'est plus où une coupure existait entre cadres «publics » et cadres "privés»; une certaine collaboration s'instaure entre eux, cependant un clivage semble subsister entre ceux que I'on nomme parfois les technocrates et qui sont du côté des « dirigeants " et la masse des ingénieurs ou des cadres autodidactes qui demeurent du côté des « dirigés».

Mais, par delà les préoccupations et les intentions des acteurs sociaux, patronat, syndicats, Etat, le sociologue est amené à se poser à l'égard du groupe des cadres des questions fondamentales qui intéressent l'évolution des structures de l'entreprise et de la société, et donc l'avenir des sociétés industrielles. 'C'est pourquoi avant même de présenter les résultats nécessairement limités d'une enquête menée auprès des ingénieurs et cadres d'une industrie de «pointe $», I^{\prime}$ 'on s'efforcera de les situer dans une problématique plus large, celle du changement: qu'est-ce qui a changé dans la situation professionnelle des cadres? Leur croissance numérique favorise-t-elle chez eux une prise de conscience collective? Si oui, quel en est le contenu et la signification? Quel est le système d'allégeance des cadres dans l'entreprise? Se considèrent-ils comme faisant partie de la direction, ou bien se considèrent-ils comme des salariés comme les autres, ou encore comme une catégorie intermédiaire et, dans certains cas, en position d'arbitre entre la direction et les ouvriers? Enfin quelle est leur stratégie? Et d'abord en ont-ils une ou plusieurs? Optent-ils délibérément pour l'action individuelle dans la course au pouvoir? Ont-ils tendance à se replier sur l'autodéfense de leur catégorie; ou bien au contraire cherchent-ils à se solidariser avec les autres catégorie de salariés? Autant de questions qui ont sans doute en commun d'être centrées sur le statut et le rôle des cadres dans l'entreprise, mais qui nécessairement renvoient à la place que tiennent les cadres dans la société et au rôle qu'ils sont amenés à y jouer.

Partant des transformations de la situation professionnelle des codres, on ne se limitero pas pour autant à les considérer dans une perspective de pure adaptation ou changement, mettant l'accent sur le déterminisme de la technique ou de l'organisation sur leurs attitudes et leurs conduites, mais on les considérera tout autant dans leur volonté de changement.

On s'interrogera alors sur les modalités et la signification de l'action collective des cadres et sur les formes de groupements, d'associations ou de syndicats par lesquels cette oction collective peut s'exprimer; tout en étant conscient que cette action collective puisse parfois traduire une résistance au changement.

Cette étude sociologique des rapports entre profession et organisation parmi les cadres jette un peu de lumière sur la position toujours discutée du personnel de cadres. De lecture assez facile, elle peut être d'un intérêt marqué pour les dirigeants d'entreprise et tous ceux qui militent dans le syndicalisme.

\section{Pierre DIONNE}

Nouveau manuel de l'agent de maîtrise, par Pierre Demarne et Jacques Ferras, Entreprise Moderne d'Edition, Paris, 1967, 157 pages.

Plusieurs personnes friandes de lecture touchant le domaine du travail connaissent 
déjò le Manuel pratique de l'agent de maîtrise » publié en 1953 par Entreprise Moderne d'Edition, réédité à huit reprises et diffusé à plus de 80,000 exemplaires. Le présent ouvrage remplace ce dernier et est conçu dans le même esprit: un instrument de formation professionnelle pour les contremaitres.

Déjà vieux de quatorze ans, l'ancien manuel devait être modernisé et mis à jour. Depuis 1953, en effet, des changements sont survenus un peu partout: de nouveaux types de relations humaines ont été créés dans le milieu social et le milieu du travail, de nouvelles méthodes d'organisations sont apparues, de nouvelles techniques voient le jour.

Partant, les auteurs se rendent compte de la nécessité pour les agents de maîtrise de connaître ces changements tant pour leur bien-être que pour celui des autres. Les ouvriers qui entrent à l'usine sont de plus en plus qualifiés et responsables; l'agent de maitrise doit donc renouveler ses connaissances et même les agrandir de façon à ne pas perdre de vue ces travailleurs.

S'il ne veut pas se retrouver moins compétent que ceux qu'il dirige, l'agent de maîtrise ne peut pas ce complaire dans ses connaissances actuelles. Regarder les gens ovec des yeux neufs, satisfaire sa curiosité intellectuelle, savoir poser des questions, avoir l'esprit éveillé, voilà un homme qui évolue ovec son temps. Tous ces besoins que peut avoir un agent de maîtrise seront en grande partie satisfaits à la lecture de ce volume qui traite de trois sujets principaux: le métier de chef, l'organisation de l'entreprise, le rôle de l'agent de maîtrise.

La première partie de l'ouvrage "Le métier de chef définit ce qu'est une entreprise, situe le commandement et donne finalement les diverses fonctions du chef selon Foyol.

Lo deuxième partie traite de l'organisation générale de l'entreprise. On y retrouve les diverses fonctions de l'entreprise décrites très brièvement exception faite de la fonction technique étudiée sous tous ses angles: la conception du produit et sa réalisation, l'aménogement et l'entretien des biens de lo société, le prix de revient.

La troisième partie analyse le rôle de l'agent de maîtrise ; il ne serait pas nécessaire de spécifier que c'est la partie la plus importante de l'ouvrage. Tous les aspects du travail de l'agent de maîtrise sont étudiés. De la nécessité de la qualité du produit au besoin, pour l'agent de maîtrise, de connaî- tre ses hommes en passant par l'outillage utilisé, la formation du personnel, la sécurité au travail, chaque fois, les auteurs lui donnent des conseils utiles et des moyens efficaces pour qu'il puisse remplir le mieux possible son rôle d'agent de maîtrise.

En plus du précieux contenu de ce manuel, soulignons la présentation attrayante de ce livre: nombreuses illustrations, impression deux couleurs, reliure à anneaux.

\section{Rosaire ROY}

\section{Ressources humaines pour le développement industriel, B.I.T., Genève, 1967, 276 pp.}

Le présent volume rassemble une série de documents qui visent à cerner quelquesuns des principoux problèmes que les pays en voie de développement rencontrent au cours de leur industrialisation dans le domaine de la main-d'oeurre et des questions sociales, ainsi qu'à examiner les mesures à prendre, aux échelons national et international, pour résoudre ces problèmes.

Les documents contenus dans ce volume sont répartis en deux groupes. La première partie, intitulée "Le problème de la maind'oeuvre ", comprend quatre études consacrées aux problèmes de la formation professionnelle et de l'utilisation de la maind'oeuvre en vue du développement industriel. Les sujets suivants, entre autres, y sont examinés:

a) détermination des buts, quantitatifs et qualitatifs, à atteindre en matière d'enseignement et de formation professionnelle de la main-d'oeuvre industrielle qualifiée ;

b) établissement de systèmes cohérents et efficaces fondés sur une base législative et administrative adéquate et création d'organisations nationales de formation industrielle, en vue d'atteindre les objectifs ainsi définis;

c) rôle particulier de l'industrie elle-même dans le perfectionnement des qualifications professionnelles;

d) mesures à prendre pour utiliser au mieux lo main-d'oeuvre qualifiée disponible ;

e) possibilités d'action sur le plan international pour permettre aux pays en voie de développement d'élaborer et de mener à bien des programmes concrets de formation professionnelle en vue de l'industrialisation.

La deuxième partie du volume, intitulée * Autres questions exigeant une politique 